Orthopäde 2009 · 38:412-418

DOI 10.1007/s00132-008-1385-3

Online publiziert: 1. Mai 2009

(c) Springer Medizin Verlag 2009

M. Beck, ${ }^{1,2}$ S.F. Fucentese ${ }^{1} \cdot$ L. Staub ${ }^{3} \cdot$ K. Siebenrock ${ }^{1}$

${ }^{1}$ Klinik für Orthopädische Chirurgie, Inselspital, Universität Bern

${ }^{2}$ Klinik für Orthopädische Chirurgie, Luzerner Kantonsspital, Luzern

${ }^{3}$ Center for Biomedical Engineering, Research Institute for Surgical Technology and Biomechanics, Universität Bern

\title{
Therapie des femoroazetabulären Impingements über die chirurgische Hüftluxation
}

\section{Technik und Ergebnisse}

\begin{abstract}
Die chirurgische Hüftluxation ist eine etablierte Technik zur Luxation der Hüfte mit einer geringen Komplikationsrate, insbesondere ohne Risiko einer avaskulären Nekrose des Femurkopfes. Damit steht eine hervorragende Methode zur Behandlung intraartikulärer Pathologien zur Verfügung. Insbesondere das femoroazetabuläre Impingement (FAI) lässt sich damit sehr präzise behandeln. Die chirurgische Hüftluxation ist deshalb zurzeit der Goldstandard der Behandlung des FAI.
\end{abstract}

Die Behandlung des FAI über die chirurgische Hüftluxation hat eine Erfolgsrate von $75-80 \%$ guten bis sehr guten Resultaten. Es konnte gezeigt werden, dass die Erhaltung des Labrums einerseits klinisch deutlich bessere Resultate ergibt als dessen Entfernung, gleichzeitig ist auch die Progression der Arthrose nach Resektion des Labrums signifikant größer. Relative Kontraindikationen sind Arthrosegrade $>1$ nach Tönnis, da hier die Ergebnisse deutlich abfallen.

\section{Technik der chirurgischen Hüftluxation}

Die Technik der chirurgischen Hüftluxation wurde erstmals von Ganz et al. [10] beschrieben und beruht auf der klinischen
Anwendung der Arbeit von Gautier et al [12], welche den exakten Verlauf der A. circumflexa femoris medialis (ACFM) in einer anatomischen Studie erforscht und beschrieben hat. Beruhend auf früheren Arbeiten von Sevitt u. Thomson [19], welche gezeigt hatten, dass die Blutzufuhr zum Hüftkopf über die ACFM alleine sichergestellt ist und auf der Arbeit von Gautier wurde eine Technik entwickelt, welche (bei korrekter Durchführung) die Luxation der Hüfte ermöglicht, ohne das Risiko einer Hüftkopfnekrose einzugehen. Dass die Durchblutung erhalten bleibt, konnte auch mittels Laserdopplermessungen nachgewiesen werden [17]. Zusätzlich zur Originalbeschreibung von Ganz et al. [10] sind zwischenzeitlich mehrere Publikationen veröffentlicht worden, welche auch die Techniken sehr detailliert beschreiben $[8,13]$. Im Folgenden soll eine Zusammenfassung des Zugangs und der intraartikulären Korrektur gegeben werden.

Die Operation erfolgt in Seitenlage, mit mobil abgedecktem Bein. Je nach Größe und Dicke des Patienten erfolgt eine ca. $20-25 \mathrm{~cm}$ lange über der Trochanterspitze zentrierte Inzision ( $\bullet$ Abb. 1). Nach Durchtrennen der Subkutis wird der Tractus iliotibialis längs gespalten. Kranial des Trochanter maior wird der Traktus entlang des Vorderrandes des M. gluteus maximus $10-12 \mathrm{~cm}$ nach proximal inzidiert. Der M. gluteus maximus wird nach dorsal weggehalten und der Hinterrand des M. gluteus medius dargestellt. Die Hüfte wird nun in Innenrotation gebracht, indem der abgewinkelte Unterschenkel auf einen etwas erhöhten Beistelltisch gelagert wird. Dadurch wird die Durchführung der Trochanterosteotomie erleichtert. Proximal endet die Trochanterosteotomie an der Spitze des Trochanter, so dass noch einige Sehnenfasern des M. gluteus medius an der stabilen Trochanterbasis verbleiben (• Abb. 2). So wird gewährleistet, dass die Osteotomie nicht in die Fossa piriformis ausläuft, was eine Durchtrennung des R. profundus der ACFM mit konsekutiver Femurkopfnekrose zur Folge hätte. Distal läuft die Osteotomie am Tuberculum inominatum aus, dem trochantären Ursprung des M. vastus lateralis.

Mit einem breiten Meißel wird der Trochanter angehoben, der $\mathrm{M}$. vastus lateralis von dorsal nach ventral vom Femur abgelöst und die Sehnenfasern des M. gluteus medius von der Trochanterbasis abgesetzt. Darunter kommt nun die Sehne und der Muskelbauch des M. piriformis zum Vorschein. In der Regel sind noch einige Fasern der Sehne des M. piriformis am Trochanterfragment, welche die Mobilisation des Trochanter nach ventral verhindern. Nach Absetzten dieser Fasern kann der Trochanter schrittweise nach ventral mobilisiert werden. Durch Außenrotati- 
on der Hüfte wird der M. piriformis entspannt. Dies ermöglicht die Präparation des Intervalls zwischen M. piriformis und M. gluteus minimus. Letzterer wird schrittweise von dorsal nach ventral von der Gelenkkapsel freipräpariert, bis er mit dem Trochanter nach ventral weggehalten werden kann. Von distal kommend, wird der M. vastus lateralis und intermedius von der Linea intertrochanterica und Gelenkkapsel freipräpariert. Die Gelenkkapsel ist nun vollständig dargestellt, auBer dem Areal, das unter dem M. piriformis liegt. Die Dissektion darf unter keinen Umständen distal der Piriformissehne erfolgen, da damit der Verlauf des R. profundus der ACFM gefährdet wird.

Die Kapsulotomie hat 3 Teile: Der 1. Teil beinhaltet einen geraden Schnitt, der etwa $1 \mathrm{~cm}$ dorsal der Spina iliaca anterior inferior, entlang des Bigelow-Ligaments zur ventralen Trochanterspitze reicht. Es muss darauf geachtet werden, dass das Labrum bei diesem Schritt nicht verletzt wird. Im 2. Schritt wird die Kapsel entlang dem Pfannenrand nach dorsal abgelöst, bis die Sehne des M. piriformis ein weiteres Präparieren nach dorsal verhindert. Im letzten Schritt wird die Kapsel entlang der Linea intertrochanterica abgelöst. Nun kann die Hüfte in allen Richtungen bewegt werden und das vermutete FAI bestätigt werden (• Abb. 3).

Das Bein wird nun in den ventral vom Patienten angebrachten sterilen Sack gelagert und durch Außenrotation und Zug an einem Haken um den Kalkar nach ventral luxiert. Die vollständige Luxation der Hüfte ist erst nach Durchtrennen des Lig. capitis femoris möglich. Nun ist ein vollständiger Überblick über Acetabulum und Hüftkopf möglich und allfällige Knorpelschäden und Labrumpathologien können erfasst und dokumentiert werden.

Nach der intraartikulären Befunderhebung wird zunächst die azetabuläre Pathologie korrigiert. Je nachdem, ob es sich um eine globale (Coxa profunda, Protrusion) oder lokalisiert vermehrte Überdachung handelt, wird entweder zirkumferentiell oder lediglich der retrovertierte Bereich abgetragen. Dort wo der Pfannenrand zurückgetrimmt werden soll, wird zunächst das Labrum scharf vom Pfannenrand abgelöst und allfällige degenerierte Teile débridiert. Der ossäre Pfannenrand wird anschließend mit einem gebogenen Meißel zurückgetrimmt. Ziel ist lateral einen Zentrum-Erker-Winkel von $25-30^{\circ}$ zu erzielen. Dies wird auf dem präoperativen Röntgenbild ausgemessen, wobei $1^{\circ}$ etwas weniger als $1 \mathrm{~mm}$ entspricht. Ausgehend vom lateralen Pfannenerker wird der hintere und vordere Pfannenrand gegen das Ende des Hinter- und Vorderhorns auslaufend abgetragen. Ziel ist eine Anteversion von $10-15^{\circ}$ zu schaffen. Das Labrum wird anschließend mit Knochenankern refixiert. Je nach Ausmaß der Pfannenrandresektion werden 2-8 Knochenanker verwendet. Ziel der Labrumrefixation ist es wieder ein Vakuumphänomen herstellen zu können.

Nun wird der Hüftkopf dargestellt. Mit Hilfe einer Schablone kann nun die Sphärizität des Hüftkopfes überprüft werden und der Beginn der Asphärizität des Hüftkopfes bestimmt werden. Oft ist nach einiger Zeit eine rötliche Verfärbung genau am Übergang vom sphärischen zum asphärischen Teil des Kopf-Hals-Übergangs festzustellen. An dieser Stelle beginnt die Resektion. Mit einem gebogenen Meißel, wird die konvexe Kontur in eine leicht konkave umgewandelt, die gegen die Linea intertrochanterica ausläuft. Tendenziell wird eher etwas zu wenig korrigiert. Die Asphärizität liegt in der Regel am ventrolateralen Schenkelhals, kann aber bis ganz lateral und/oder bis ventral lokalisiert sein [6]. Die kritischste Stelle, um eine Korrektur durchzuführen, liegt unmittelbar hinter der 12-Uhr-Position des Schenkelhalses, dort wo die retinakulären Aste des R. profundus der ACFM im Bereich des "soft spots“ in die Epiphyse des Hüftkopfes eintreten (• Abb. 4). Jede Beschädigung dieser Blutgefäße würde eine Hüftkopfnekrose nach sich ziehen. Wenn hier eine Korrektur durchgeführt wird, muss die Technik den anatomischen Gegebenheiten angepasst werden, indem mit dem Meißel der asphärische Teil partiell osteotomiert, anschließend ausgebrochen und schließlich subperiostal ausgeschält wird. Vor der Reposition der Hüfte, wird das Lig. capitis femoris von der Fovea reseziert. Die impingementfreie Beweglichkeit wird in allen Richtungen überprüft. Angestrebt wird eine Innenrotation von $20-25^{\circ}$ der $90^{\circ}$ gebeugten Hüfte. Wird dies nicht erreicht, wird die Hüfte noch-
Orthopäde 2009 · 38:412-418

DOI 10.1007/s00132-008-1385-3

(C) Springer Medizin Verlag 2009

M. Beck - S.F. Fucentese - L. Staub - K. Siebenrock

Therapie des femoroazetabulären Impingements über die chirurgische Hüftluxation. Technik und Ergebnisse

Zusammenfassung

Die chirurgische Hüftluxation ist eine sichere und etablierte Technik für die Behandlung des femoroazetabulären Impingements. Die Komplikationsrate ist niedrig und mit der korrekten Technik, welche die Blutversorgung respektiert, tritt eine Femurkopfnekrose nicht auf. Die häufigsten Komplikationen sind milde ektope Ossifkationen und die Trochanterpseudarthrose. Die intraartikuläre Chirurgie schließt sowohl die azetabuläre wie auch femorale Korrektur ein. Klinisch kann in ca. $75-80 \%$ der Fälle ein gutes bis sehr gutes Resultat erzielt werden. Allerdings fällt die Erfolgschance beim Vorliegen fortgeschrittener degenerativer Veränderungen, welche eine Grad-1-Arthrose nach Tönnis überschreiten, rapide ab. Der Erhalt des Labrums hat einen signifikanten Einfluss auf das klinische Ergebnis und die radiologische Progression der Arthrose. Das Erhalten des Labrums scheint deshalb unabdingbar zu sein.

Schlüsselwörter

FAI - Chirurgische Hüftluxation · Technik .

Resultate $\cdot$ Labrum

Surgical dislocation of the hip for the treatment of femoroacetabular impingement. Technique and results

\section{Abstract}

Surgical dislocation of the hip is a safe and established technique for treating femoroacetabular impingement. The complication rate is low, and if the correct technique that respects the blood supply is used, femoral head necrosis does not occur. The most frequent complications are minor ectopic bone formation and nonunion of the greater trochanter. Surgical treatment includes the correction of femoral and acetabular pathology. Clinically, in approximately $75-80 \%$ of cases a good-to-excellent result can be obtained. However, patients with advanced degenerative changes (exceeding stage 1 osteoarthritis using the Tönnis score) have worse outcomes. It has also been shown that preservation of the labrum has a significant influence on both clinical outcome and progression of osteoarthritis: It seems that preservation of the labrum is mandatory.

Keywords

FAI - Surgical dislocation · Technique - Results · Labrum 


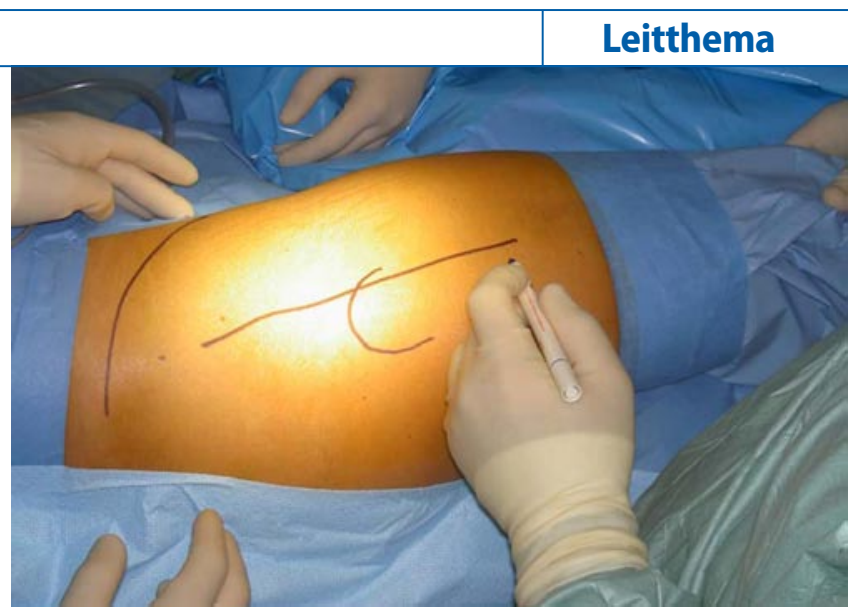

Abb. $1<$ Patient in Linksseitenlage, rechte Hüfte oben: Die Inzision ist auf den kranialen Rand des Trochanters zentriert und verläuft im vorderen Drittel des Trochanters

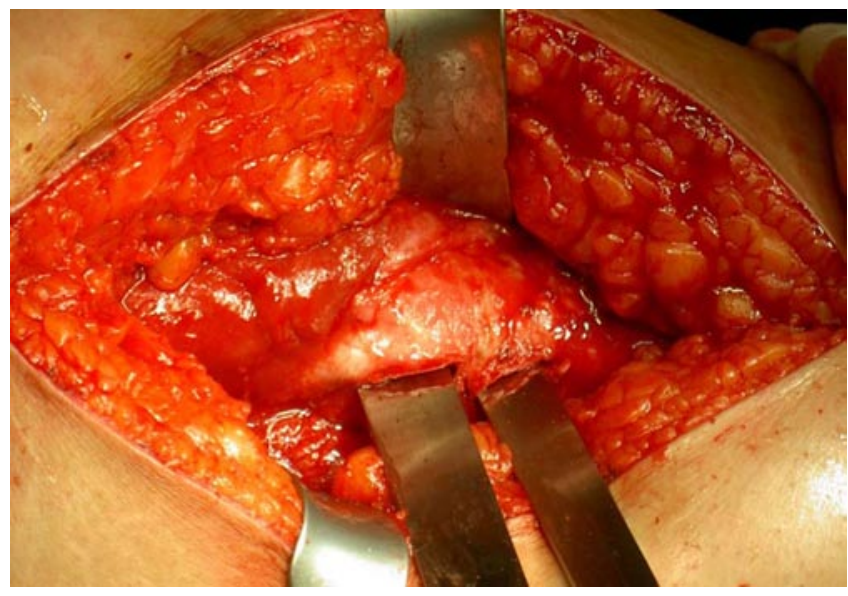

Abb. $2<$ Stufenosteotomie des Trochanters: Anstelle der flachen Osteotomie kann eine Stufenosteotomie durchgeführt werden, um die Stabilität der Refixation zu erhöhen. Damit kann bereits nach 4 Wochen zur Vollbelastung übergegangen werden und Einschränkungen des postoperativen Bewegungsumfangs entfallen

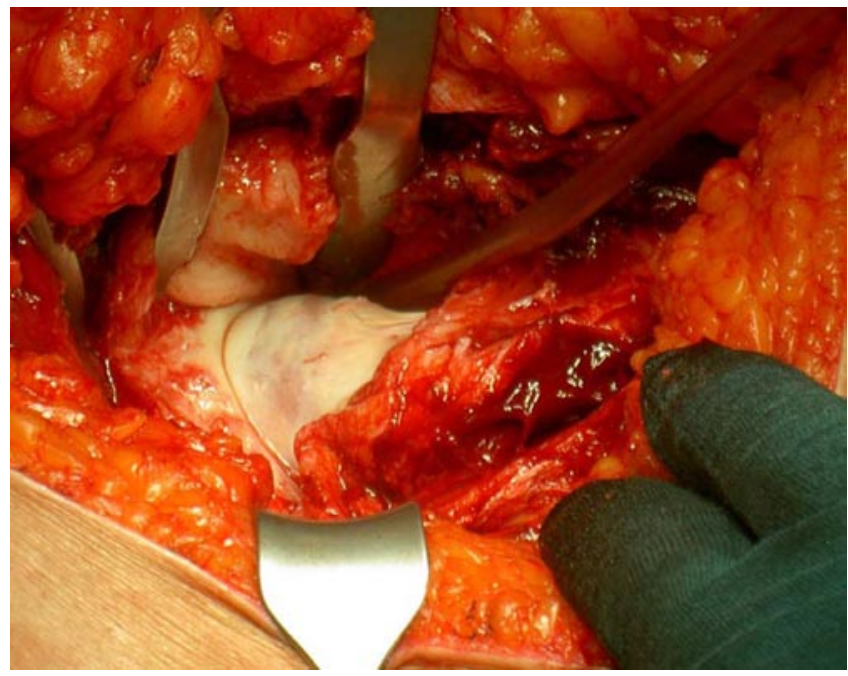

Abb. $3 \varangle$ Rechte Hüfte nach Osteotomie des Trochanters und Kapsulotomie: Die Asphärizität des KopfHals-Übergangs ist bereits gut sichtbar. Beim Durchbewegen der Hüfte kann das FAl beobachtet werden mals luxiert und der Schenkelhals an entsprechender Stelle nachgetrimmt.

Die Kapsel wird locker verschlossen und der Trochanter mit zwei 3,5-mmKortikalisschrauben verschraubt. Die Gleitschichten über dem Trochanter major werden ebenfalls locker adaptiert und die Inzision nach der Naht des Tractus iliotibialis schichtweise verschlossen. Die Nachbehandlung ist einerseits durch die Trochanterosteotomie limitiert, anderer-

\section{Hier steht eine Anzeige.}

\author{
Springer
}

seits durch die Notwendigkeit intraartikuläre Verklebungen zu verhindern.

Während den ersten 6 Wochen postoperativ wird die Hüfte passiv bis $90^{\circ}$ durchbewegt und die Mobilisation mit einer Teilbelastung von $10-15 \mathrm{~kg}$ erlaubt. Nach erfolgter Konsolidation der Trochanterosteotomie erfolgt ein rascher Belastungsaufbau mit einer physiotherapeutisch geführten intensiven Rehabilitation. Entsprechend sind leichte sportliche Akti- 
Hier steht eine Anzeige.

算 Springer 


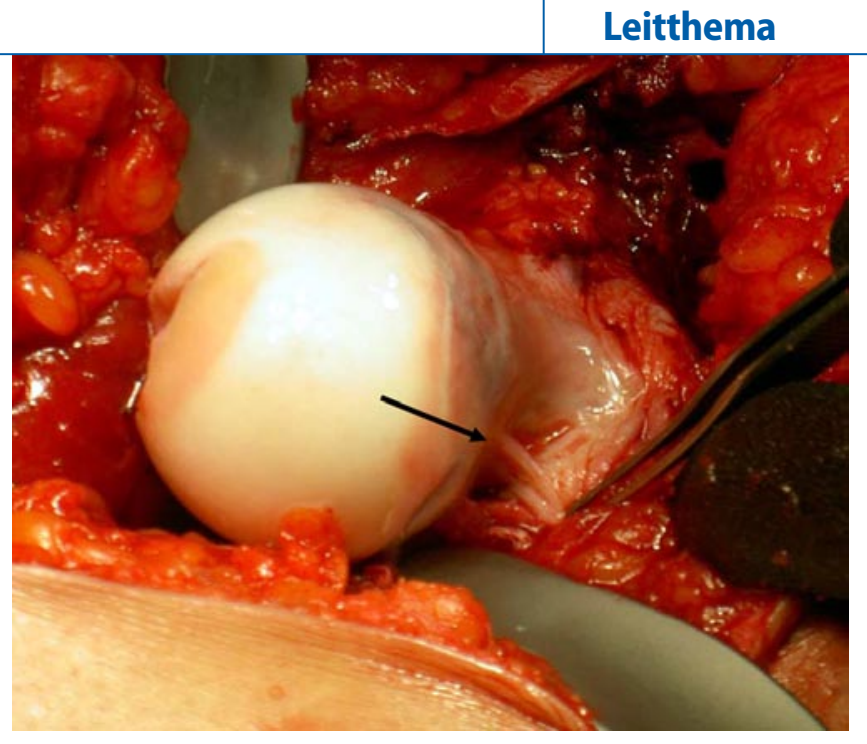

Abb. $4<$ Luxierter Hüftkopf: Das Lig. capitis femoris ist bereits reseziert worden. In der Synovialfalte (Pfeil) verlaufen die Endäste $\operatorname{der}$ A. circumflexa femoris medialis

vitäten etwa 3 Monate postoperativ möglich. Mit intensivem Training kann etwa 46 Monate postoperativ begonnen werden.

\section{Ergebnisse}

Ganz et al. [10] publizierten die Technik der chirurgischen Hüftluxation an $213 \mathrm{~Pa}$ tienten. Insgesamt ist die Komplikationsrate sehr gering. Eine Femurkopfnekrose trat nicht auf. In 2 Fällen trat eine Neurapraxie des N. ischiadicus auf, die sich innerhalb eines halben Jahres spontan erholte. In beiden Fällen bestand ein Zustand nach vorgängiger Operation und möglicherweise waren Vernarbungen um den N. ischiadicus, welche die Geleitfähigkeit beeinträchtigten, dafür verantwortlich. Eine Trochanterpseudarthrose trat in 3 Fällen und klinisch signifikante ektope Ossifikationen in 2 Fällen auf. In beiden Fälle führte die Entfernung zur Verbesserung der Beweglichkeit. Insgesamt waren ektope Ossifikationen an der Trochanterspitze häufig, überschritten jedoch Brooker-I-Stadien nur sehr selten.

Das FAI ist eine gesicherte Ursache der mechanisch bedingten Hüftarthrosen, welches insbesondere schon beim jungen Patienten symptomatisch wird [11]. Neben klinischen Arbeiten [2] gibt es auch mehrere biomechanische Untersuchungen, welche den Effekt des FAI auf das Hüftgelenk nachweisen $[5,7,20]$. Aktuell gibt es nur wenige Publikationen über die Ergebnisse der Behandlung des FAI über die chirurgische Hüftluxation. Die erste Publikation stammt von Beck et al. [3] und umfasste 19 Fälle. Das mittlere Alter der
14 Männer und 5 Frauen betrug 36 (2152) Jahre. Das Follow-up war im Mittel 4,7 (4,0-5,2) Jahre. Der Merle-d'AubignéScore verbesserte sich signifikant von 14,1 auf 16,5 Punkte. In 14 (73\%) Hüften war das Resultat gut bis exzellent. Eine Femurkopfnekrose trat nicht auf. Fünf der $19 \mathrm{~Pa}$ tienten (2 mit einem Arthrosegrad 2, 2 mit einem Arthrosegrad 1 aber ausgedehnten azetabulären Knorpelschäden und einer Hüfte, bei der die Pincerkomponente nicht behandelt wurde) benötigten im Verlauf die Implantation einer Hüftprothese. Ein schlechtes klinisches Ergebnis korrelierte mit dem azetabulären Knorpelschaden zum Zeitpunkt der Operation. Die Autoren schlussfolgerten, dass Hüften mit Arthrosegrad >1 und/oder fortgeschrittenen azetabulären Knorpelschäden für diese Art gelenkerhaltender Chirurgie nicht geeignet sind.

Ähnliche Ergebnisse wurden von Murphy et al. [16] publiziert. In ihrer Gruppe von 23 Patienten hatten $65 \%$ ein gutes bis exzellentes Resultat, in 7 Fällen musste eine Hüftprothese implantiert werden. Der Grund dafür waren fortgeschrittene degenerative Veränderungen mit einem Arthrosegrad 2-3. Auch Beaulé et al. [1] berichteten über vergleichbare Resultate. In einer Gruppe von 34 Patienten (37 Hüften) wurde eine Verbesserung sowohl des WOMAC, als auch des UCLA-ActivityScores und des SF12-Scores gesehen. Eine Femurkopfnekrose wurde nicht beschrieben und keine Hüfte war zum Zeitpunkt der Studie zu einer Endoprothese konvertiert worden. Die Autoren kamen zum Schluss, dass die chirurgische Hüftluxa- tion mit Osteochondroplastik des Schenkelhalses eine sichere Methode darstellt und eine signifikante Verbesserung der Lebensqualität mit sich bringt. Peters et al. [18] berichteten über 30 Hüften in $29 \mathrm{~Pa}$ tienten mit FAI. Der Harris-Hip-Score (HHS) verbesserte sich im Mittel von 70 auf 87 Punkte nach einem Mindestnachuntersuchungszeitraum von 2 Jahren. Femurkopfnekrosen wurde keine beobachtet, ebenso keine Trochanterpseudarthrose. Bei 18 Patienten wurden intraoperativ schwere azetabuläre Knorpelschäden entdeckt, was aufgrund der präoperativen Abklärungen nicht erwartet worden war. Von diesen hatten 8 in der Folge eine progressive Gelenkspaltverschmälerung und 4 mussten schließlich mit einer Hüftprothese versorgt werden.

In einer retrospektiven Studie verglichen Espinosa et al. [9] den Einfluss der Labrumrefixation. Dabei wurde eine Gruppe von 25 Patienten, bei denen im Rahmen der Pfannenrandtrimmung das Labrum reseziert wurde mit 35 Hüften verglichen, bei denen das Labrum nach der Pfannenrandtrimmung wieder refixiert wurde. Es zeigte sich dabei, dass die Hüften mit der Labrumrefixation nicht nur klinisch signifikant besser waren (94\% gut - exzellent), sondern nach 2 Jahren auch eine geringere Zunahme der Arthroserate aufwiesen. Die Autoren zogen die Schlussfolgerung, dass der Erhalt des Labrums unabdingbar sei.

Obwohl das FAI oft bei professionellen oder ambitionierten Hobbysportlern diagnostiziert und behandelt wird, gibt es kaum Publikationen über die Ergebnisse bei Hochleistungssportlern. Eine Ausnahme macht die Arbeit von Bizzini et al. [4], der die 2-Jahres-Ergebnisse bei 5 professionellen Eishockeyspielern zusammenfasste. Dabei wurden v. a. die Sportfähigkeit und die funktionelle Erholung der Hüftmuskulatur untersucht. Die Ergebnisse sind vielversprechend. Die Kraft der Becken- und Hüftmuskulatur erreichte präoperative Werte nach einem Mittel von 7,8 Monaten. Uneingeschränktes Training konnte nach 6,7 Monaten und Wettkämpfe nach 9,6 Monaten wiederaufgenommen werden. Drei der 5 Spieler erreichten das gleiche Niveau wie vor der Operation.

In einer eigenen retrospektiven Studie versuchten wir prädiktive Faktoren zu 
Hier steht eine Anzeige.

算 Springer 
identifizieren, die zu einem schmerzfreien (d. h. exzellenten) Ergebnis führen. Da radiologisch im Zeitraum von 5 Jahren keine Abnahme des Gelenkspalts nachweisbar ist [3], wurde auf die radiologische Nachuntersuchung verzichtet und an 315 Patienten ein Fragebogen verschickt. Dieser Fragebogen diente ursprünglich dazu, den allgemeinen Gesundheitszustand der durchschnittlichen Schweizer Bevölkerung zu eruieren und ist validiert [15]. 277 Fragebogen wurden zurückgeschickt (Rücklaufrate 88\%) und ausgewertet. Die Gruppe umfasste 47\% Frauen, 53\% Männer mit einem Durchschnittsalter von 32,5 (13-66) Jahren. Die mittlere Nachsorgezeit betrug 4 (1-6) Jahre. In $17 \%$ lag ein Cam-, in $3 \%$ ein Pincer- und in $80 \%$ ein gemischtes Cam-Pincer-Impingement vor. Die degenerativen Veränderungen wurden nach Tönnis klassifiziert [21] und waren wie folgt verteilt: Grad o 10\%, Grad 1 59\%, Grad 2 29\% und Grad 3 1\%. Der klinische Verlauf wurde mit dem Merle-d'Aubigné- (MDA-)Score gemessen. Insgesamt wurden ca. 90 klinische und radiologische Parameter erfasst und mittels multivariater Regressionsanalyse analysiert; u. a. Alter, Geschlecht, Impingement-Typ, präoperativer MDAScore und präoperativer Arthrosegrad. Der MDA-Score verbesserte sich im Mittel von 14,3 auf 16,3 Punkte.

Insgesamt lag in $87 \%$ ein gutes bis sehr gutes Resultat vor $(\mathrm{MDA}>16)$. Der Schmerzscore verbesserte sich bei $61 \%$ und bliebt bei $22 \%$ konstant. Interessant ist $\mathrm{zu}$ sehen, dass v. a. die Patienten mit einem präoperativen Schmerzscore von 4 oder 5 am häufigsten auf dem gleichen Schmerzniveau blieben, während v. a. die Patienten darunter am meisten vom Eingriff profitierten. Vor allem die Persistenz eines Leistenschmerzes schlug sich am stärksten negativ auf das klinische Ergebnis aus. Die Regressionsanalyse ergab eine signifikante Korrelation für den Arthrosegrad [Odds-Ratio $(\mathrm{OR})=0,38$ ], männliches Geschlecht $(\mathrm{OR}=1,88)$, Cam-Impingement $(\mathrm{OR}=3,14)$, Merle-d'Aubigné-Score $(\mathrm{OR}=1,36)$ und zweite Hüfte $(\mathrm{OR}=2,1)$. Das bedeutet, dass jede Zunahme des Arthrosegrades um 1 Punkt die Chance für ein schmerzfreies Resultat um den Faktor 3 vermindert. Jeder zusätzliche Punkt im MDA-Score lässt die Wahrscheinlich- keit für eine schmerzfreie Hüfte um den Faktor 1,3 steigen. Das männliche Geschlecht hat fast die doppelte Chance auf eine schmerzfreie Hüfte als das weibliche, das Cam-FAI die 3 fache Chance im Vergleich zum gemischten FAI und die zweite Hüfte die doppelte Chance für ein schmerzfreies Ergebnis.

Zusammenfassend kann man daraus schließen, dass die besten Ergebnisse der FAI-Behandlung bei männlichen $\mathrm{Pa}$ tienten mit einem Cam-Impingement, einem Arthrosegrad von $\mathrm{O}$ und einem hohem MDA-Score zu erzielen sind.

\section{Fazit für die Praxis}

Die chirurgische Hüftluxation ist eine sichere und komplikationsarme Technik zur Behandlung des femoroazetabulären Impingements. Die Vorteile liegen in der exzellenten Übersicht über das ganze Hüftgelenk und die Präzision, mit der die notwendigen Korrekturen durchgeführt werden können. Bei Anwendung der korrekten Technik sind bisher keine Femurkopfnekrosen beschrieben worden. Die klinischen Ergebnisse der Behandlung des FAl über die chirurgische Hüftluxation sind relativ konstant und ein gutes bis sehr gutes Resultat kann in $\mathbf{7 5 - 8 0 \% ~ d e r ~}$ Patienten erzielt werden. Voraussetzung dazu ist jedoch das Fehlen radiologischer Zeichen der Gelenkdegeneration. Die Erfolgsrate nimmt bei Arthrosegraden $>1$ nach Tönnis rapide ab.

\section{Korrespondenzadresse \\ PD Dr. M. Beck \\ Klinik für Orthopädische Chirurgie, \\ Luzerner Kantonsspital \\ CH-6000 Luzern, Schweiz \\ martin.beck@ksl.ch}

Interessenkonflikt. Der korrespondierende Autor gibt an, dass kein Interessenkonflikt besteht.

\section{Literatur}

1. Beaulé PE, Le Duff MJ, Zaragoza E (2007) Quality of life following femoral head-neck osteochondroplasty for femoroacetabular impingement. J Bon Joint Surg Am 89: 773-779

2. Beck M, Kalhor M, Leunig M, Ganz R (2005) Hip morphology influences the pattern of damage to the acetabular cartilage: Femoroacetabular impingement as a cause of early osteoarthritis of the hip. J Bone Joint Surg Br 87: 1012-1018
3. Beck M, Leunig M, Parvizi J et al (2004) Anterior femoroacetabular impingement. Part II: Midterm results of surgical treatment. Clin Orthop Relat Res 418: 67-73

4. Bizzini M, Nötzli HP, Maffiuletti NA (2007) Femoroacetabular impingement in professional ice hockey players. A case series of 5 athletes after open surgical decompression of the hip. Am J Sports Med 35: 1955-1959

5. Chegini S, Beck M, Ferguson SJ (2009) The effects of impingement and dysplasia on stress distributions in the hip joint during sitting and walking: $A$ finite element analysis. J Orthop Res 27: 195-201

6. Dudda M, Albers C, Mamisch TC et al (2009) Do normal radiographs exclude asphericity of the femoral head-neck junction? Clin Orthop Relat Res 467(3): 651-659

7. Ito K, Minka MA, Leunig M et al (2001) Femoroacetabular impingement and the cam-effect: An MRI based quantitative study of the femoral headneck offset. J Bone Joint Surg Br 83: 171-176

8. Espinosa N, Beck M, Rothenfluh DA et al (2007) Treatment of femoro-acetabular impingement: Preliminary results of labral refixation. Surgical technique. J Bone Joint Surg Am 89(Suppl 2): 36-53

9. Espinosa N, Rothenfluh DA, Beck M et al (2006) Treatment of femoro-acetabular impingement: Preliminary results of labral refixation. J Bone Joint Surg Am 88: 925-935

10. Ganz R, Gill TJ, Gautier E et al (2001) Surgical dislocation of the adult hip: A technique with full access to the femoral head and acetabulum without the risk of avascular necrosis. J Bone Joint Surg Br 83: 1119-1124

11. Ganz R, Parvizi J, Beck M et al (2003) Femoroacetabular impingement: a cause for osteoarthritis of the hip. Clin Orthop Relat Res 417: 112-120

12. Gautier E, Ganz K, Krugel N et al (2000) Anatomy of the medial femoral circumflex artery and its surgical implications. J Bone Joint Surg Br 82: 679-683

13. Lavigne M, Parvizi J, Beck M et al (2004) Anterior femoroacetabular impingement: Part I. Techniques of joint preserving surgery. Clin Orthop Relat Res 418: 61-66

14. Merle d'Aubigné R, Postel M (1954) Functional results of hip arthroplasty with acrylic prosthesis. J Bone Joint Surg Am 36: 451-475

15. Müller U, Tänzler K, Bürger A et al (2008) A pain assessment scale for population-based studies: Development and validation of the pain module of the standard evaluation questionnaire. Pain 136: 62-74

16. Murphy S, Tannast M, Kim YJ et al (2004) Debridement of the adult hip for femoroacetabular impingement: Indications and preliminary clinical results. Clin Orthop Relat Res 429: 178-181

17. Nötzli HP, Siebenrock KA, Hempfing A et al (2002) Perfusion of the femoral head during surgical dislocation of the hip: Monitoring by laser doppler flowmetry. J Bone Joint Surg Br 84: 300-304

18. Peters CL, Erickson JA (2006) Treatment of femoroacetabular impingement with surgical dislocation and débridement in young adults. J Bone Joint Surg Am 88:1735-1741

19. Sevitt S, Thompson RG (1965) The distribution and anastomoses of arteries supplying the head and neck of the femur. J Bone Joint Surg Br 47: 560-573

20. Tannast M, Goricki D, Beck M et al (2008) Hip damage occurs at the zone of femoroacetabular impingement. Clin Orthop Relat Res 466: 273-280

21. Tönnis D (1976) Normal values of the hip for the evaluation of $x$-rays in children and adults. Clin Orthop Relat Res 119: 39-47 